\title{
Kidney disease: new technologies translate mechanisms to cure
}

\author{
John F. O'Toole ${ }^{1}$ and John R. Sedor',2 \\ ${ }^{1}$ MetroHealth System and Department of Medicine and 2Department of Physiology and Biophysics, Rammelkamp Center for Education and Research, \\ Case Western Reserve University, Cleveland, Ohio, USA.
}

\begin{abstract}
Kidney disease is one of the most prevalent chronic conditions and is a frequent complication of diabetes, cardiovascular disease, and obesity. Recent advances in biomedical research and novel technologies have created opportunities to study kidney disease in a variety of platforms, applied to human populations. The Reviews in this series discuss the kidney in hypertension, diabetes, and monogenic forms of kidney disease, as well as the cellular and molecular mediators of acute kidney injury and fibrosis, IgA nephropathy and idiopathic membranous nephropathy, and kidney transplantation. In this introduction, we briefly review new insights into focal segmental glomerulosclerosis and the role of podocytes in health and disease. Additionally, we discuss how new technologies, therapeutics, and the availability of patient data can help shape the study of kidney disease and ultimately inform policies concerning biomedical research and health care.
\end{abstract}

\section{The burden of kidney disease}

The advent of a uniform definition and staging of kidney disease radically altered our understanding of its impact on human health (1). Prior to this, only kidney disease that required replacement therapy was recognized as a significant health issue. The medical and scientific communities viewed kidney dysfunction, although devastating to the unfortunate affected individuals, as rare and of minimal consequence to population health. With uniform definitions, we now know that kidney disease is common. The Centers for Disease Control and Prevention estimates that over 20 million Americans have chronic kidney disease (CKD), including more than $40 \%$ of individuals older than 65 years (2). Global CKD prevalence is estimated to be greater than $10 \%$ in many countries $(1,3)$. CKD is a frequent complication of chronic diseases that are prevalent in the United States, including diabetes, obesity, hypertension, and cardiovascular disease. Additionally, the presence of CKD is associated with significantly more adverse outcomes for patients with cardiovascular disease and diabetes compared with individuals without CKD. CKD therapy is expensive: in 2010 the U.S. Medicare program spent $\$ 32.9$ billion providing treatment for 690,000 individuals with end-stage renal disease (ESRD), which accounted for more than $7 \%$ of Medicare expenses attributed to less than $1 \%$ of Medicare beneficiaries. Even in early stage CKD, estimates of added Medicare costs are substantial (4). CKD patients frequently progress to ESRD despite widespread adoption of optimal renoprotective treatments (5-8).

Despite these stark realities, investigators studying kidney biology and disease have a stunning history of creatively addressing the burden of kidney disease. Examples include the use of kidney biopsy to guide diagnosis and treatment, the development of renal replacement modalities, and pioneering work in transplantation that directly resulted in at least one Nobel Prize. An unintended but welcome result has been life-saving diagnostics and therapies that benefit patients with some diseases unrelated to the kidney. For example, efforts to effectively treat the anemia of ESRD spawned erythropoietin, perhaps the most successful drug to result from recombinant DNA technology (9).

Conflict of interest: The authors have declared that no conflict of interest exists. Citation for this article: J Clin Invest. 2014;124(6):2294-2298. doi:10.1172/JCI76825.
The last decade has witnessed the emergence of novel technologies that will lead investigators to the next major breakthrough. As these technologies become increasingly available, generating higher data density at lower costs, their utility as tools for discovery has been leveraged. These methods often were initially optimized for use with cell culture and animal models, but now are also being utilized to directly study biological samples from human populations, permitting deep phenotyping and linkage with clinical data collected in electronic health records. For the first time, we have access to samples from large numbers of affected patients, which permits discovery of distinct molecular taxonomies that have been undetectable using current experimental diagnostic modalities. Many seminal papers now start with discovery using "-omics" datasets from patients and subsequently move to the bench to define the mechanisms of action. Often these studies also begin the path back to the bedside, with leads for novel biomarkers or drugs. These advances in the biological sciences, coupled with the increasing availability of clinical data afforded by the implementation of electronic medical records and advances in imaging modalities, have catalyzed advances in the cause, diagnosis, and treatment of kidney disease.

For this series, we invited Reviews that encompass the breadth of nephrology research, avoiding focus on a single biological theme, in order to reflect the excitement engendered by discoveries in different areas of kidney biology and disease. A number of these Reviews discuss use of new technologies to test key questions and show their impact on our understanding of kidney disease pathogenesis, which will affect prevention, prognosis, and treatment. In this introductory article, we first highlight key points of these Reviews, focusing on the commonality of themes. We then discuss other recent discoveries in the field of kidney disease, which also demonstrate the rapid advances in therapies that promise restoration of health - a cure for our patients, rather than a life of debility, dialysis, and death (10).

Common traits: diabetes and bypertension. Diabetes and hypertension are two common complex diseases and remain the most frequent causes of ESRD in the United States, accounting for over 70\% of incident cases between 2007 and 2011 (1, 11). Reciprocal kidney transplantation studies in rodent models have shown that hyper- 
tension susceptibility or resistance was transmitted with the transplanted organ (12-15), which supported Arthur Guyton's hypothesis that kidney dysfunction is the cause of elevated blood pressure (16). Subsequent studies used genetic approaches in rodent models to selectively remove genes encoding the components of the reninangiotensin-aldosterone system (RAAS) from defined tissues (17, 18). In this Review series, Steve Crowley and Tom Coffman review their pioneering studies, which showed that intrarenal RAAS function controls blood pressure and provides a molecular explanation for the earlier cross-species transplantation experiments $(19,20)$. While ongoing research on kidney RAAS and blood pressure regulation continues to build on and amplify these observations, other studies highlight additional, surprising mechanisms. Although the kidney clearly plays a central role in blood pressure control, Crowley and Coffman discuss the unexpected and interesting work that demonstrated hypertonic accumulation of sodium in the subdermal space and its contribution to blood pressure control (20-22). Another recent study presents intriguing data showing that blocking of NHE3-dependent sodium uptake in the intestine of animal models of hypertension improves extracellular fluid volume and reduces blood pressure and cardiac hypertrophy (23).

Kidney disease remains a devastating complication of diabetes. In cohorts of both type 1 and type 2 diabetic patients, the presence and severity of diabetic kidney disease (DKD) explains much of the excess mortality attributed to diabetes (24-27). Despite widespread adoption of renoprotective interventions, almost $50 \%$ of the macroalbuminuric type 1 diabetic patients followed in Boston and Finland progressed to ESRD $(6,7)$. In addition, landmark clinical trials and observational cohort studies have documented the limitations of current therapies for DKD, showing that further intensification of glycemic and blood pressure control beyond current guidelines does not confer additional benefit and may even cause harm $(28,29)$. Counterintuitively, even early blockade of the RAAS failed to slow nephropathy progression in type 1 diabetic patients who were not hypertensive and had normal albumin excretion (30). These studies indicate the need for new treatment targets that will only be developed with a better understanding of DKD pathogenesis.

The definition of underlying genetic architecture has been critical to advances in other diseases (31). Multicenter collaborative efforts utilizing unbiased genome-wide association studies (GWAS) of large cohorts have yielded the first insights into the genetic contributors of the initiation and progression of DKD (32-34). The biological pathways modified by the genetic variants underlying diabetic nephropathy remain unclear. Similar to GWAS of other disease phenotypes, variants associated with diabetic nephropathy are in non-protein coding regions of the genome. The functional significance of disease variants identified by GWAS has been debated (35); they have been discounted as statistical artifacts or, alternatively, proposed to be proxy SNPs for true, but perhaps rare, causal variants within the exome (36). However, recent data indicate that GWAS associations are not random and are located in regions bounded by recombination hot spots near non-coding causal variants that regulate transcription $(31,37,38)$. These genetic alterations may change the epigenetic (chemical) modifications of DNA and histones required for normal, tissue-specific gene expression (39). In her Review, Katalin Susztak explores the hypothesis that metabolic dysregulation in diabetic subjects causes epigenetic reprogramming that drives the progressive diabetic nephropathy phenotype (40). Clinical data support this hypothesis. The Diabetes Control and Complications Trial/Epidemiology of Diabetes Interventions and Complications (DCCT/EDIC) study demonstrated that the incidence of diabetic nephropathy remained greater in subjects with standard versus intensive glycemic control during a 5-year interventional trial despite excellent glycemic control in both groups during the subsequent 20 -year observational phase $(41,42)$. Epigenetic changes may be the mechanism behind this long-term "metabolic memory" of glycemic control. The relationship between genetic and epigenetic variation in common disease has pushed the development of epigenome-wide association studies, which can be designed as independent analyses or integrated with GWAS in order to discover pathways that drive disease phenotypes $(39,43,44)$.

\section{Polycystic kidney disease: "bench" strength drive clinical trials}

Autosomal dominant polycystic kidney disease (ADPKD) represents the most common monogenetic cause of ESRD, accounting for an additional $2 \%$ of all incident ESRD cases between 2007 and 2011 (11). Genetic studies continue to inform phenotypic variation and extra-renal manifestations, while proteinbased studies identify downstream pathways and provide therapeutic targets. Animal models continue to have an important role in the study of cyst formation, where an emerging concept is the idea that a threshold level of polycystin activity exists, below which cystogenesis occurs $(45,46)$. The downstream pathways and emerging treatment strategies are featured in the Review of ADPKD by Peter Harris and Vicente Torres (47). The recognition of dysregulated energy metabolism in models of ADPKD has led to the identification of aerobic glycolysis as a novel treatment target $(48,49)$. Interactions between leukocytes and kidney cells are highlighted in several of the Reviews (see below), and a role of macrophages in cystogenesis has also been identified. This mechanism explains previous reports that germ-free conditions prevent cyst growth and also suggests that ADPKD pathogenesis may share some mechanistic similarities with acute kidney injury $(50,51)$.

\section{Cross-talk between infiltrating leukocytes and kidney cells: clues to acute kidney injury and kidney fibrogenesis}

Macrophages as well as other elements of the immune system are emerging as important mediators of acute kidney injury (52) and the fibrotic components of the wound healing response (53). These Reviews also highlight the complexity of interactions between endogenous kidney cells in the initiation of kidney injury and the mechanisms that underlie renal fibrosis. In the setting of acute kidney injury, Bruce Molitoris discusses how the interaction between proximal tubular cells and local endothelial cells results in the local alteration of microvascular circulation through the recruitment of innate immune molecules and circulating white blood cells (52). Jeremy Duffield presents his overarching view that kidney fibrosis is a wound-healing response that persists, contributing to deposition of matrix material between tubules and peritubular vasculature, impairing exchange of waste and nutrients between capillary and tubular lumens, and reinforcing tubular damage and progressive loss of renal function (53). Fatemapping studies show that pericytes and resident fibroblasts arise from FOXD1 lineage precursors and, in disease settings, undergo 
differentiation into myofibroblasts that perpetuate inflammation and recruit leukocytes from the circulation $(54,55)$. Other studies have suggested a more diverse origin for these cells (56). Characterization of myofibroblasts has identified several signaling pathways enriched or restricted to FOXD1 lineage cells that may permit specificity in developing therapies for fibrogenesis $(57,58)$

\section{Podocytopathy discoveries: specific mechanisms uncover tools for clinical utility}

Important advances have been made in our understanding of the pathogenetic mechanisms that contribute to the common glomerular diseases IgA nephropathy (IgAN), reviewed by Krzysztof Kiryluk and Jan Novak (59), and idiopathic membranous nephropathy, reviewed by Laurence Beck and David Salant (60). These advances have been informed by studies using animal models and investigations in human populations. While the pathogenic details of each disease are unique, they do share some common themes. The first is the idea that genetic variants contribute to disease susceptibility. GWAS have implicated aberrant antigen processing and presentation for both IgAN and membranous nephropathy, and have identified variants in mucosal immunity and alternative complement pathways for IgAN and in PLA2R1 for membranous nephropathy. These data suggest that aberrant antigen processing and presentation contribute to the pathogenesis of both diseases. Second, in both diseases, nephritogenic antibodies, which result from abnormal antigen processing, deposit in the glomerulus and cause tissue injury in both IgAN and membranous nephropathy. Knowledge of this has led to the development of testing strategies that hold promise for improving the diagnosis and treatment of individuals with these diseases. Early studies suggest that PLA2R1 antibodies predict outcome and response to treatment in membranous nephropathy (61). Work is ongoing to assess diagnostic or predictive biomarkers in IgAN based on the discovery that IgA1 is underglycosylated in patients with IgAN compared with healthy subjects without disease (62).

\section{Complement and kidney allograft injury: opportunities for intervention}

One of the most effective therapies for the treatment of ESRD remains kidney transplantation. Extending graft survival is an important goal, since studies have demonstrated lower mortality (63) and higher quality of life (64) with transplantation compared with dialysis. The role of complement activation as a mediator of graft rejection and long-term graft survival is reviewed in this issue by Paolo Cravedi and Peter Heeger (65). Animal studies have suggested that complement factors elaborated by cells resident in the transplanted organ have an important role in mediating ischemia-reperfusion kidney injury (66). Complement cascade activity of the recipient is also an important determinant of the immune response and ultimately graft survival. These data point to blocking complement cascade activity as an important target in further improving outcomes in kidney transplant patients.

\section{Other recent discoveries move the needle closer to a cure for kidney diseases}

Mutations in $U M O D$, which encodes uromodulin, were well known as the genetic etiology of medullary cystic kidney disease type 2, a rare autosomal dominant Mendelian kidney disease (67). Subsequent unbiased GWAS have found UMOD variants in association with glomerular filtration rate in populations with European ancestry (68, $69)$, but the mechanism underlying this association has remained obscure. Recent work showed that $U M O D$ risk variants are associated with an increase in $U M O D$ expression and hypertension, which is dependent upon the NKCC2 sodium/potassium/chloride transporter (70). These results provide a link between CKD and hypertension and suggest a strategy for the rational selection of medications. Subjects with the UMOD risk variants and CKD may be more effectively treated with loop diuretics as first-line therapy rather than thiazide diuretics, in stark contrast to current guidelines, which are based on large hypertension trials that have excluded individuals with CKD.

A landmark discovery associated genetic variations in the APOL1 gene with excess prevalence of advanced, non-diabetic CKD in African Americans $(71,72)$, and recent data expanded the association to include diabetic CKD (8). Although APOL1 kidney disease-associated variants are common (12\% of African Americans carry two risk alleles), only a subset of these individuals develop advanced CKD (72-74), suggesting that a "second hit" is required for disease initiation. APOL1 mediates trypanosome killing and prevents African sleeping sickness $(75,76)$. A subspecies of trypanosome produces serum resistance associated (SRA) protein, which binds APOL1 and blocks its trypanolytic activity (77). APOL1 coding polymorphisms, G1 and G2, circumvent SRAdependent resistance and restore parasite killing but, if present on both alleles, strongly increase CKD risk (71). Our recent studies showed that APOL1 is localized in kidney cells (78) and that the G1 and G2 variants do not change APOL1 localization, but likely alter its function. Kidney allograft outcomes are associated with the APOL1 genotype of the transplanted kidney but not the recipient $(79,80)$, and we have shown that circulating levels of APOL1 do not associate with CKD phenotypes (81), together suggesting that APOL1 within kidney mediates the associated CKD.

Two other stories are developing in the study of focal segmental glomerulosclerosis (FSGS) that center on molecular drivers of disease and their role in podocyte health and disease. The first is an observation that implicates microRNAs in the disease pathogenesis of FSGS. Using a mouse model of miRNA 193a (miR-193a) overexpression, investigators found that these animals develop FSGS through the downregulation of WT1, a governor of the transcriptional program in podocytes (82). These results were followed with human studies demonstrating that miR-193a is also increased in the glomeruli of individuals with FSGS and may contribute to the pathogenesis of FSGS through a similar mechanism.

Another example is illustrated by the accumulating in vitro and in vivo evidence supporting a role for the expression of B7-1 (CD80) in the podocyte as an important contributor to proteinuria as well as a potential therapeutic target. Lipopolysaccharide treatment of murine models induces proteinuria and expression of B7-1, and B7-1 knockout animals are protected from proteinuria, which supports a causal role for B7-1 in podocytopathic glomerular disease (83). B7-1 expression has been observed in a spectrum of renal pathologies (84) and may exert its pathogenic effect through the modulation of $\beta 1$-integrin activation and cell motility. A recent report also demonstrates that high glucose concentrations is able to induce B7-1 expression in podocytes, possibly extending the association from a spectrum of rare glomerular disease to DKD, which is the most common cause of glomerular pathology and ESRD in the United States (85). In the setting of hyperglycemia, B7-1 expression is mediated by a PI3K-mediated mechanism (85); whether this mechanism is relevant to other glomerular pathologies remains to be investigated. Abatercept is an antibody against B7-1 and is FDA approved for treatment of rheumatoid arthritis 
(86). Five patients with FSGS resistant to current therapies and with increased B7-1 expression on kidney biopsy achieved a complete or partial remission after treatment with abatercept (84). These results need to be tested in a randomized controlled trial.

Integration of basic science, translational, and clinical studies is needed to address the serious obstacles to providing effective care for kidney disease patients. The Nephrotic Syndrome Study Network (NEPTUNE) provides a successful model to achieve this outcome. NEPTUNE has recruited a cohort of patients with FSGS, idiopathic membranous nephropathy, and minimal change disease, and has generated data sets that define the underlying genetic architecture and capture environmental exposures, unique molecular phenotypes, and histopathology linked to prospective clinical outcomes. This disease knowledge along the genotype-phenotype continuum is being used to define nephrotic syndrome from its molecular pathogenesis rather than histopathological patterns $(87,88)$.

\section{Conclusion}

CKD, like many complex disease phenotypes, reflects dynamic interactions between genetic susceptibilities and environmental factors. Unraveling the relative contributions and interactions of our genome and epigenome, diet and activity, and exposure to microorganisms and medications will be the major challenge in the near future. While it is easy to see that at this moment in time medicine is poised to change dramatically, what it will look like in the future is far more difficult to discern. However, the future of medicine will be shaped by our imagination as we create new technologies and creatively apply them to improve the health of individuals with kidney diseases. CKD is common and life limiting, but progress in understanding its mechanisms, often catalyzed by discovery using human biosamples and new technologies, makes its cure more tangible and the future of nephrology bright.

\section{Acknowledgments}

J.F. O'Toole and J.R. Sedor gratefully acknowledge support from the NIH NIDDK (grants DK097836, DK083912, DK100846, UL1TR000439, and DK091597) and the MetroHealth System.

Address correspondence to: John R. Sedor, 2500 MetroHealth Drive R415, Cleveland, Ohio 44109-1998, USA. Phone: 216.778.4993; Fax: 216.778.8248; E-mail: john.sedor@case.edu.
1. Levey AS, Coresh J. Chronic kidney disease. Lancet. 2012;379(9811):165-180.

2. CDC. 2014 National Chronic Kidney Disease Fact Sheet. Atlanta, Georgia, USA: US Department of Health and Human Services, Centers for Disease Control and Prevention; 2014

3. Eckardt KU, et al. Evolving importance of kidney disease: from subspecialty to global health burden. Lancet. 2013;158(9887):158-169.

4. Honeycutt AA, Segel JE, Zhuo X, Hoerger TJ, Imai K, Williams D. Medical costs of CKD in the Medicare population. J Am Soc Nephrol. 2013;24(9):1478-1483.

5. Lipkowitz MS, et al. Apolipoprotein L1 gene variants associate with hypertension-attributed nephropathy and the rate of kidney function decline in African Americans. Kidney Int. 2012; 83(1):114-120.

6. Forsblom C, et al. Competing-risk analysis of ESRD and death among patients with type 1 diabetes and macroalbuminuria. J Am Soc Nephrol. 2011;22(3):537-544.

7. Rosolowsky ET, et al. Risk for ESRD in type 1 diabetes remains high despite renoprotection. J Am Soc Nephrol. 2011;22(3):545-553.

8. Parsa A, et al. APOL1 risk variants, race, and progression of chronic kidney disease. $N$ Engl J Med. 2013;369(23):2183-2196.

9. Bunn HF. Erythropoietin. Cold Spring Harb Perspect Med. 2013;3(3):a011619.

10. Falk RJ. ASN presidential address 2012: Time to cure kidney disease. J Am Soc Nephrol. 2013; 24(7):1040-1043.

11. Atlas of chronic kidney disease and end-stage renal disease in the United States. In: USRDS 2013 Annual Data Report. Bethesda, Maryland, USA: National Institutes of Health, National Institute of Diabetes and Digestive and Kidney Diseases; 2013.

12. Kawabe K, Watanabe TX, Shiono K, Sokabe H. Influence on blood pressure of renal isografts between spontaneously hypertensive and normotensive rats, utilizing the F1 hybrids. Jpn Heart J. 1978;19(6):886-894.

13. Dahl LK, Heine M. Primary role of renal homografts in setting chronic blood pressure levels in rats. Circ Res. 1975;36(6):692-696.

14. Bianchi G, Fox U, Di Francesco GF, Giovanetti AM, Pagetti D. Blood pressure changes produced by kidney cross-transplantation between spontaneously hypertensive rats and normotensive rats. Clin Sci Mol Med. 1974;47(5):435-448.
15. Dahl LK, Heine M, Thompson K. Genetic influence of the kidneys on blood pressure. Evidence from chronic renal homografts in rats with opposite predispositions to hypertension. Circ Res. 1974;40(4):94-101.

16. Guyton AC. The surprising kidney-fluid mechanism for pressure control - its infinite gain! Hypertension. 1990;16(6):725-730.

17. Crowley SD, et al. Angiotensin II causes hypertension and cardiac hypertrophy through its receptors in the kidney. Proc Natl Acad Sci U S A. 2006;103(47):17985-17990.

18. Gonzalez-Villalobos RA, et al. The absence of intrarenal ACE protects against hypertension. J Clin Invest. 2013;123(5):2011-2023.

19. Coffman TM, Crowley SD. Kidney in hypertension: guyton redux. Hypertension. 2008;51(4):811-816.

20. Crowley SD, Coffman TM. The inextricable role of the kidney in hypertension. J Clin Invest. 2014;124(6):2341-2347.

21. Wiig H, et al. Immune cells control skin lymphatic electrolyte homeostasis and blood pressure. J Clin Invest. 2013;123(7):2803-2815.

22. Titze J, et al. Spooky sodium balance. Kidney Int. 2014;85(4):759-767.

23. Spencer AG, et al. Intestinal inhibition of the $\mathrm{Na}+/$ $\mathrm{H}+$ exchanger 3 prevents cardiorenal damage in rats and inhibits $\mathrm{Na}+$ uptake in humans. Sci Transl Med. 2014;6(227):227ra36.

24. Groop PH, et al. The presence and severity of chronic kidney disease predicts all-cause mortality in type 1 diabetes. Diabetes. 2009;58(7):1651-1658.

25. Orchard TJ, Secrest AM, Miller RG, Costacou T. In the absence of renal disease, 20 year mortality risk in type 1 diabetes is comparable to that of the general population: a report from the Pittsburgh Epidemiology of Diabetes Complications Study. Diabetologia. 2010;53(11):2312-2319.

26. de Boer IH, Rue TC, Hall YN, Heagerty PJ, Weiss NS, Himmelfarb J. Temporal trends in the prevalence of diabetic kidney disease in the United States. JAMA. 2011;305(24):2532-2539.

27. Afkarian $M$, et al. Kidney disease and increased mortality risk in type 2 diabetes. J Am Soc Nephrol. 2013;24(2):302-308.

28. Ismail-Beigi $F$, et al. Effect of intensive treatment of hyperglycaemia on microvascular outcomes in type 2 diabetes: an analysis of the ACCORD randomised trial. Lancet. 2010;376(9739):419-430.

29. Gerstein HC, et al. Long-term effects of intensive glucose lowering on cardiovascular outcomes. NEngl J Med. 2011;364(9):818-828.

30. Mauer M, et al. Renal and retinal effects of enalapril and losartan in type 1 diabetes. $N$ Engl J Med. 2009;361(1):40-51.

31. Green ED, Guyer MS. Charting a course for genomic medicine from base pairs to bedside. Nature. 2011;470(7333):204-213.

32. Sandholm N, et al. New susceptibility loci associated with kidney disease in type 1 diabetes. PLoS Genet. 2012;8(9):e1002921.

33. Igo RP, et al. Genomewide linkage scan for diabetic renal failure and albuminuria: the FIND study. $A m$ J Nephrol. 2011;33(5):381-389.

34. Pezzolesi MG, et al. Genome-wide association scan for diabetic nephropathy susceptibility genes in type 1 diabetes mellitus. Diabetes. 2009;58(6):1403-1410.

35. Manolio TA, et al. Finding the missing heritability of complex diseases. Nature. 2009;461(7265):747-753.

36. Goldstein DB. The importance of synthetic associations will only be resolved empirically. PLoS Biol. 2011;9(1):e1001008.

37. Lango AH, et al. Hundreds of variants clustered in genomic loci and biological pathways affect human height. Nature. 2010;467(7317):832-838.

38. Gu Y, et al. Long-term renal changes in the liverspecific glucokinase knockout mouse: implications for renal disease in maturity-onset diabetes of the young 2. Transl Res. 2011;157(3):111-116.

39. Edwards SL, Beesley J, French JD, Dunning AM. Beyond GWASs: illuminating the dark road from association to function. Am J Hum Genet. 2013; 93(5):779-797.

40. Reidy K, Kang HM, Hostetter T, Susztak K. Molecular mechanisms of diabetic kidney disease. J Clin Invest. 2014;124(6):2333-2340.

41. Writing Team for the Diabetes Control Complications Trial/Epidemiology of Diabetes Interventions Complications Research Group. Sustained effect of intensive treatment of type 1 diabetes mellitus on development and progression of diabetic nephropathy: the Epidemiology of Diabetes Interventions and Complications (EDIC) study. JAMA. 2003;290(16):2159-2167.

42. de Boer IH, et al. Long-term renal outcomes of patients with type 1 diabetes mellitus and microalbuminuria: an analysis of the Diabetes Control and Complications Trial/Epidemiology of Diabetes Interventions and Complications cohort. Arch Intern Med. 2011;171(5):412-420. 
43. Callaway E. Epigenomics starts to make its mark. Nature. 2014;508(7494):22.

44. Murphy TM, Mill J. Epigenetics in health and disease: heralding the EWAS era. Lancet. 2014; pii:S0140-6736(14)60269-5

45. Hopp K, et al. Functional polycystin-1 dosage governs autosomal dominant polycystic kidney disease severity. J Clin Invest. 2012;122(11):4257-4273.

46. Gallagher AR, Germino GG, Somlo S. Molecular advances in autosomal dominant polycystic kidney disease. Adv Chronic Kidney Dis. 2010;17(2):118-130.

47. Harris PC, Torres VE. Genetic mechanisms and signaling pathways in autosomal dominant polycystic kidney disease. J Clin Invest. 2014;124(6):2315-2324.

48. Wang $X$, et al. Targeting of sodium-glucose cotransporters with phlorizin inhibits polycystic kidney disease progression in Han:SPRD rats. Kidney Int. 2013;84(5):962-968.

49. Rowe I, et al. Defective glucose metabolism in polycystic kidney disease identifies a new therapeutic strategy. Nat Med. 2013;19(4):388-393.

50 . Lee $\mathrm{S}$, et al. Distinct macrophage phenotypes contribute to kidney injury and repair. J Am Soc Nephrol. 2011;22(2):317-326.

51. Ricardo SD, van Goor H, Eddy AA. Macrophage diversity in renal injury and repair. J Clin Invest. 2008;118(11):3522-3530.

52. Molitoris BA. Therapeutic translation in acute kidney injury: the epithelial/endothelial axis. J Clin Invest. 2014;124(6):2355-2363.

53. Duffield JS. Cellular and molecular mechanisms in kidney fibrosis. J Clin Invest. 2014;124(6):2299-2306.

54. Duffield JS. Macrophages in kidney repair and regeneration. J Am Soc Nephrol. 2011;22(2):199-201.

55. Lichtnekert J, Kawakami T, Parks WC, Duffield JS. Changes in macrophage phenotype as the immune response evolves. Curr Opin Pharmacol. 2013; 13(4):555-564

56. Lebleu VS, et al. Origin and function of myofibroblasts in kidney fibrosis. Nat Med. 2013; 19(8):1047-1053

57. Meyers CM, Briggs JP. Silymarin for diabetic nephropathy: the challenges of botanical product research. Am J Kidney Dis. 2012;60(6):887-889.

58. Friedman SL, Sheppard D, Duffield JS, Violette S Therapy for fibrotic diseases: nearing the starting line. Sci Transl Med. 2013;5(167):167sr1.

59. Kiryluk K, Novak J. The genetics and immunobiology of IgA nephropathy. J Clin Invest. 2014 124(6):2325-2332.
60. Beck LH Jr, Salant DJ. Membranous nephropathy: from models to man. J Clin Invest. 2014, 124(6):2307-2314.

61. Hoxha E, Thiele I, Zahner G, Panzer U, Harendza S, Stahl RA. Phospholipase A2 receptor autoantibodies and clinical outcome in patients with primary membranous nephropathy [published online ahead of print March 7, 2014]. J Am Soc Nephrol. doi:10.1681/ASN.2013040430.

62. Hastings MC, et al. Biomarkers in IgA nephropathy: relationship to pathogenetic hits. Expert Opin Med Diagn. 2013;7(6):615-627.

63. Wolfe RA, et al. Comparison of mortality in all patients on dialysis, patients on dialysis awaiting transplantation, and recipients of a first cadaveric transplant. N Engl J Med. 1999;341(23):1725-1730.

64. Wyld M, Morton RL, Hayen A, Howard K, Webster AC. A systematic review and meta-analysis of utility-based quality of life in chronic kidney disease treatments. PLoS Med. 2012;9(9):e1001307.

65. Cravedi P, Heeger PS. Complement as a multifaceted modulator of kidney transplant injury. J Clin Invest. 2014;124(6):2348-2354

66. Farrar CA, Zhou W, Lin T, Sacks SH. Local extravascular pool of $\mathrm{C} 3$ is a determinant of postischemic acute renal failure. FASEB J. 2006;20(2):217-226.

67. Hart TC, et al. Mutations of the UMOD gene are responsible for medullary cystic kidney disease 2 and familial juvenile hyperuricaemic nephropathy. J Med Genet. 2002;39(12):882-892.

68. Kottgen A, et al. Multiple loci associated with indices of renal function and chronic kidney disease. Nat Genet. 2009;41(6):1191-1198.

69. Kottgen A, et al. New loci associated with kidney function and chronic kidney disease. Nat Genet. 2010;42(5):376-384.

70. Trudu M, et al. Common noncoding UMOD gene variants induce salt-sensitive hypertension and kidney damage by increasing uromodulin expression. Nat Med. 2013;19(12):1655-1660.

71. Genovese G, et al. Association of trypanolytic ApoL1 variants with kidney disease in African Americans. Science. 2010;329(5993):841-845.

72. Tzur S, et al. Missense mutations in the APOL1 gene are highly associated with end stage kidney disease risk previously attributed to the MYH9 gene. Hum Genet. 2010;128(3):350.

73. Baker P, Broughton Pipkin F. Screening tests for pregnancy-induced hypertension. Prog Obstet Gynaecol. $1993 ; 10(5$ pt 1):69-86.
74. Kopp JB, et al. APOL1 genetic variants in focal segmental glomerulosclerosis and HIV-associated nephropathy. J Am Soc Nephrol. 2011;22(11):2129-2137.

75. Perez-Morga D, et al. Apolipoprotein L-I promotes trypanosome lysis by forming pores in lysosomal membranes. Science. 2005;309(5733):469-472.

76. Vanhamme L, et al. Apolipoprotein L-I is the trypanosome lytic factor of human serum. Nature. 2003;422(6927):83-87.

77. Pays E, Vanhollebeke B, Vanhamme L, PaturiauxHanocq F, Nolan DP, Perez-Morga D. The trypanolytic factor of human serum. Nat Rev Microbiol. 2006;4(6):477-486

78. Madhavan SM, O'Toole JF, Konieczkowski M, Ganesan S, Bruggeman LA, Sedor JR. APOL1 localization in normal kidney and nondiabetic kidney disease. J Am Soc Nephrol. 2011;22(11):2119-2128.

79. Reeves-Daniel AM, et al. The APOL1 gene and allograft survival after kidney transplantation. Am J Transplant. 2011;11(5):1025-1030.

80. Lee BT, et al. The APOL1 genotype of African American kidney transplant recipients does not impact 5-year allograft survival. Am J Transplant. 2012;12(7):1924-1928.

81. Bruggeman LA, et al. Plasma apolipoprotein L1 levels do not correlate with CKD. J Am Soc Nephrol. 2013;25(3):634-644.

82. Gebeshuber CA, et al. Focal segmental glomerulosclerosis is induced by microRNA-193a and its downregulation of WT1. Nat Med. 2013;19(4):481-487.

83. Reiser J, et al. Induction of B7-1 in podocytes is associated with nephrotic syndrome. J Clin Invest. 2004;113(10):1390-1397.

84. Yu CC, et al. Abatacept in B7-1-positive proteinuric kidney disease. NEnglJMed. 2013;369(25):2416-2423.

85 . Fiorina $\mathrm{P}$, et al. Role of podocyte B7-1 in diabetic nephropathy [published online ahead of print March 27, 2014]. J Am Soc Nephrol. doi:10.1681/ ASN.2013050518.

86. Genovese MC, et al. Abatacept for rheumatoid arthritis refractory to tumor necrosis factor $\alpha$ inhibition. N Engl J Med. 2005;353(11):1114-1123.

87. Gadegbeku CA, et al. Design of the Nephrotic Syndrome Study Network (NEPTUNE) to evaluate primary glomerular nephropathy by a multidisciplinary approach. Kidney Int. 2013;83(4):749-756.

88. Barisoni L, et al. Digital pathology evaluation in the multicenter Nephrotic Syndrome Study Network (NEPTUNE). Clin J Am Soc Nephrol. 2013;8(8):1449-1459. 